\title{
3
}

\section{Men, Alcohol and Coping}

\section{Introduction}

Lord Stephen Taylor of Harlow, speaking in the House of Lords in 1965, recalled that he once knew a French GP who was 'much mystified by the English disease of the "nervous breakdown"'. The friend had observed: 'We do not have this in France. En France c'est l'alcoholisme (In France it is alcoholism)'. ${ }^{1}$ By the mid 1960s, concerns about alcohol abuse among industrial workers emerged in a number of international studies about psychological illness, driven largely, as the previous chapter has illustrated, by concerns about sickness absence in industry. A study of Australian male telegraphists, for example, drew explicit attention to the inter-relationship between sickness absence, drinking, gastritis and peptic ulcer. Drawing a direct association between drinking and neurosis, the author argued that the subsequent 'physical consequences of drinking to excess no doubt contributed to the liability of the drinker to be absent repeatedly'. ${ }^{2}$ As with much of the research on this topic, nonetheless, there was no clear consensus when it came to deciding whether the alcohol abuse was caused initially by the worker's constitution, or by the pressures of any personal or professional problems he might be experiencing. Research papers from the Netherlands articulated similar difficulties. A follow-up study of male alcoholics undertaken by clinicians at a treatment centre in Groningen proposed that troubles and conflicts in the marital and family sphere were usually present in patients; however, these conflicts were 'dependent on the pathological drinking - either being caused by it or, if present before, being intensified by it'. ${ }^{3}$ In Britain, even less was known about the antecedents of drinking behaviours, and debates about alcohol took much longer to develop. Despite clear evidence that men were more likely to 
present (and take time off work sick) with somatic symptoms, such as gastritis and peptic ulcer, often exacerbated by the use of alcohol, few investigators sought to explore the extent to which men self-medicated with alcohol for the relief of depression and emotional release. This chapter examines the complex clinical, social and cultural forces that influenced debates about alcohol abuse in Britain from the 1950s and it suggests that historically, the failure to examine drinking as a 'coping mechanism' in men has had important implications for the broader interpretation of patterns of psychological illness.

\section{Reflections on alcoholism}

The disease concept of alcoholism that became dominant during the post-war period had its roots much earlier in the late eighteenth- and nineteenth-century theories put forward simultaneously by America's Benjamin Rush (1746-1813) and Britain's Thomas Trotter (1760-1832). Their theories are now well known and broadly describe the central characteristics of alcoholism that are still familiar to us today: namely, 'powerlessness' over the substance and the 'progressive' nature of the illness. By the turn of the twentieth century, the 'disease' of inebriety had begun to find its way into medical textbooks and academic psychiatry. ${ }^{4}$ In Britain, the Society for the Study of Addiction to Alcohol and other Drugs (formed originally in 1884 as the Society for the Study and Cure of Inebriety), emphasised a medical, materialist conception of disease, despite its original aim to pursue a social medicine and public health approach. As Berridge notes, initial developments were a product of the particular state of the medical profession during a period in which physicians were, for the first time, treating 'specific' diseases with 'specific' treatments with some success. It seemed, therefore, 'only natural to extend this disease formulation to other conditions', such as homosexuality, insanity, alcoholism and drug addiction. ${ }^{5}$ The central theme of the society was 'the crusading advocacy of a disease theory of inebriety to what was seen as an outmoded, moralistic approach' and its membership 'lay firmly in the medical sphere'. ${ }^{6}$ In promoting alcoholism as a disease as opposed to a vice, the society lobbied to secure state legislation and a medical treatment structure. ${ }^{7}$ A brief change of focus followed during the First World War, when concerns about efficiency during wartime prompted discussions about the control of alcohol more broadly. Pre-war discussions had been notable for not focussing on licensing laws and other 'non-medical legislative aspects of the drink question'. ${ }^{8}$ During the inter-war period, nonetheless, these 
concerns receded and debates refocused on alcoholism as a racial and eugenic concern - although following the developments in psychiatry related to war neurosis in soldiers, there was limited and cautious acceptance in some circles of a psychological aspect to addiction. ${ }^{9}$ For a number of reasons, a major shift took place in the mid-twentieth century towards a disease model of alcohol addiction, requiring medical treatment. ${ }^{10}$ Berridge has shown that a strong biomedical emphasis developed and flourished in the post-Second World War period due to a new scientific optimism and faith in technology, which bolstered the belief in the power of clinical medicine. Simultaneously, the efficacy of psychological methods had been questioned as the process was increasingly viewed as 'tedious and long-drawn out'. ${ }^{11}$ During the 1950s, those working within the field argued that the state should play a greater role in the provision of hospital-based treatment for alcoholism; however, there was very little funding available for alcoholism research. ${ }^{12}$ By the 1960s, as this chapter will illustrate, concerns had prompted the development of a number of competing organisations, such as the National Council on Alcoholism, which was established in 1962, and the Medical Council of Alcoholism, which was formed in 1967.13

The American biostatistician and physician, Elvin Morton Jellinek (1890-1963), published his seminal piece 'Phases of Alcohol Addiction' in 1952 in which he highlighted the notion of 'loss of control' which progressed through a set of stages towards 'rock bottom' ${ }^{14}$ These principles were further developed by the German-born neurologist, Max Glatt (1912-2002), into a 'U shaped' chart depicting a 'slippery slope' with an upward path to recovery. ${ }^{15}$ In the 1970s, the British psychiatrist, Griffith Edwards (1928-2012), who became an internationally renowned expert on addiction, coined the term 'alcohol dependence syndrome', which was incorporated in the World Health Organization's International Classification of Diseases (ICD) in 1979. Griffith outlined the dependence syndrome in an article published in the British Medical Journal in 1976, co-written with American psychiatrist Milton M. Gross. ${ }^{16}$ Edward's influence on addiction studies was manifest in a prolific range of publications directed at both academic and popular readerships. ${ }^{17}$

The model of alcoholism eventually adopted by the NHS, and influential during the period under study, was that based on the work of Max Glatt at his therapeutic treatment unit at Warlingham Park, Middlesex during the 1950s. ${ }^{18}$ Although there was increasing acceptance of the notion of alcoholism as a 'disease', developments in policy and treatment in Britain were nonetheless fragmented and piecemeal. While some articulated increasing concern about alcohol abuse, there was still widespread 
denial of the problem. The first branch of Alcoholics Anonymous (AA) was founded in London in 1948 but aroused little interest among those in the medical profession. ${ }^{19}$ It is testimony to the disregard of the medical profession that three years later, in 1951, a consultant psychiatrist applied for funds to attend a World Health Organization conference on alcoholism to find that his application was rejected, on the grounds that 'there was no alcoholism in England and Wales'. ${ }^{20}$ Glatt, who first came across alcoholics when working as a psychiatrist at Warlingham Park hospital, recalled that when he became interested in alcoholism during the early 1950s, he knew 'not a thing about it' and that 'nothing much was written' about it in Britain. ${ }^{21}$ His treatment unit became a model for others that were eventually opened under the NHS and he often received foreign clinicians to his unit who came to learn about his treatment methods. Despite increasing concern about alcoholism in specialist circles, the Ministry of Health continued to deny outright that alcohol was a problem at all in England and Wales. ${ }^{22}$

In the scant statistical evidence that emerged in figures from in-patient units and general practice, men were significantly overrepresented. However, prior to the 1970s there was no organised discussion about gender in British debates about alcoholism; it was simply noted to be less common in women. Efforts instead focused upon establishing an accurate national estimate of alcoholics and discussion centred otherwise on how best to treat the condition once diagnosed. The Rowntree Steering Group on Alcoholism, set up in 1956 under the chairmanship of W. B. Morrell from the Rowntree Trust, was particularly concerned with finding a true estimate of numbers affected by alcohol abuse, since numbers varied greatly in existing studies. Jellinek had developed a formula for estimating the percentage of alcoholics in the general population based broadly on the number of deaths from liver cirrhosis in a given year. However, Denis Parr, then a Research Fellow at the Department of Psychiatry, St. George's Hospital in London, put forward a much lower estimate based on numbers presenting in general practice. ${ }^{23}$ Glatt was critical of Parr's research, arguing that GPs were not always likely to detect the early stages of alcoholism and he raised concerns that this lower estimate would increase the general apathy about alcohol abuse. ${ }^{24}$ The steering group called upon the assistance of social agencies, such as health visitors and probation officers, eventually confirming that much hidden alcoholism existed in the community, thus calling into question Parr's figures. ${ }^{25}$

Other initiatives developed along similar lines. Griffith Edwards, inspired by alcoholism programmes he had seen in America, began 
discussions during the early 1960s with a group of interested individuals in the Camberwell area of London - a move that developed into the Camberwell Council on Alcoholism (CCA). This group consisted of members drawn from medicine and psychiatry, the clergy, the police, social services and the Chamber of Commerce and it worked to educate doctors and other interested parties. While Glatt's treatment unit tended to treat middle-class drinkers, the CCA was particularly concerned about the plight of 'skid row' alcoholics and habitual drunken offenders. ${ }^{26}$ It went on to become nationally influential, in part because of the lack of other strong policy-relevant interest groups in the alcohol arena. ${ }^{27}$ Although their objective was 'to gauge the extent of the problem and to investigate personal, social and economic factors concerned in the causes of alcoholism', discussion tended to be dominated instead by its 'impact upon the life of the nation', in particular the deleterious social consequences of alcoholism: crime, social disturbance and family breakdown. ${ }^{28}$ Alcohol abuse clearly appeared to affect men in much larger numbers than women, but nonetheless, discussions rarely mentioned why this might be. Rare individual accounts from alcoholics themselves demonstrate widespread denial and reluctance to confront the problem. One former alcoholic whose contribution was published in the Journal of Alcoholism, for example, recalled that none of his friends, work colleagues or his employer ever took him aside and spoke seriously to him. Instead, he noted that they 'all connived in covering up ... what now appears to be serious drinking bouts and their attendant hangovers' ${ }^{29}$ This man declared that the situation within which he found himself was simply 'part of the rich pageant of life as [he knew] it', and he concluded that, where alcohol was concerned, he was just 'slightly more blind in a whole kingdom of the partially sighted' ${ }^{30}$

\section{General medicine}

Although researchers eventually acknowledged that much problem drinking remained unreported in the community, the official figures that existed by 1950 suggested that alcohol consumption in Britain was comparatively low. ${ }^{31}$ This contributed to the official view from the Ministry of Health that alcohol abuse was 'not a problem'. However, as Thom has shown, a number of other factors framed the discourse on alcohol abuse. Firstly, the power of the temperance movement had waned considerably and thus policy action, when it came, focused on the medical aspects of alcoholism and not on preventative measures. Secondly, the general disarray of mental health services following the 
introduction of the NHS resulted in a lack of resources for alcohol treatment. Thirdly, and perhaps most importantly, the disease model of alcoholism legitimised medicine's role in treating the condition, viewing it as a 'disease of the unfortunate minority' ${ }^{32}$ As such, debates did not focus in any serious way on the social factors and life stressors that might have contributed to individual drinking habits, nor did they address the strong cultural forces that prevented men from discussing their problems and seeking help. Indeed, the Ministry of Health was explicitly concerned about limiting their enquiries strictly to treatment issues, since prevention would open 'very wide vistas', which were thought to be quite outside the scope of the department'. ${ }^{33}$

Accounts from those working in medicine certainly reflected this approach. Casualty doctors noted that cases of alcoholism usually presented at the 'emergency end of the disease', and, because patients were admitted to general hospitals, not psychiatric wards, as soon as they were 'physically well' they were discharged. ${ }^{34}$ The emphasis on the physical nature of the condition was widely evident in accounts from hospital doctors who contributed to a series of seminars on the topic held by the CCA in 1967. One remarked, for example, that alcoholics rarely presented in 'such a mental state' that it would justify compulsory detention under Section 25 of the Mental Health Act. ${ }^{35}$ During a subsequent seminar in 1970, the Registrar in charge of Casualty at King's College Hospital similarly described his experience of treating intoxicated patients:

Should someone present himself as very depressed, we try and find a physical reason to account for this ... such as an overdose of drugs ... or some overwhelming disease - I wouldn't spend too long on it. If it's an acute problem, we treat them, but if it's not, then they have to go. Overdose is seen as a psychiatric emergency - alcoholics are not. ${ }^{36}$

The remaining seminar discussion focused on the physical treatments that were available such as stomach irrigation for alcohol poisoning and the use of vitamin injections. 'True' psychiatric cases, one doctor pointed out, were assured a consultation at the Maudsley Hospital; however, he cautioned that the broad remit was 'to find out what is the matter with him, to assess whether he should be chucked out or kept in' ${ }^{37}$ This approach was in many ways at odds with the official approach of the psychiatric profession and the classification of 'alcoholism', which was placed firmly under the heading 'Neurosis, personality disorders and other non-psychotic mental disorders', in 
the International Classification of Diseases. ${ }^{38}$ While psychiatrists were more likely to consider that alcohol problems might be related to personality disorders and neurosis, clinicians working within general medicine, often dealing with late-stage alcohol problems as emergencies, highlighted its organic and physical effects. ${ }^{39}$ This approach was also in marked contrast to the attitudes of alcohol experts such as Glatt who, although not underestimating the importance of personality, emphasised the 'great influence of social problems on the causation and development of alcoholism' ${ }^{40}$ His position was that alcoholism was both a 'symptom' and a 'disease'; 'family strife may have been caused by the drinking but [was] in itself later a cause for further drinking' ${ }^{41}$ Indeed, one of his methods of treatment involved patients telling their life-stories - a technique he had developed previously when working with neurosis patients. ${ }^{42}$ Glatt also worked closely with AA and claimed his methods complemented those employed by the organisation. ${ }^{43}$ However, despite his notable influence, the eventual development of alcohol treatment units between the early 1960s and the 1980s was slow and patchy and treatment methods were diverse. ${ }^{44}$ Glatt noted that he faced considerable inertia and that 'many doctors and professionals [were] only too keen to avoid involvement with alcoholic patients'. ${ }^{45}$ Although some provision was made for women, those who were referred to treatment units were predominantly male, likely to be in their forties and from the higher social classes. 'Skid row' drinkers were less likely to call upon services provided, and consultants were less likely to admit them to in-patient wards. Thom notes that this demographic remained stable until the 1980s. ${ }^{46}$

During the early 1970s, a small group within the CCA put forward a proposal to investigate women alcoholics. Although numbers of women were thought to be very small at a ratio with men of one to four, a review of the literature suggested that there were some specific concerns - among them the fact that within the family unit, women were usually the primary carers of children, and the fact that 'drinking at home' featured much more regularly, making it harder to detect. ${ }^{47}$ The nature of this investigation is particularly illuminating. In many ways concerns clearly reflected long-established moralistic overtones about women and alcohol. As others have shown, in the alcohol arena the focus has historically been 'not so much on women as women, but on women as mothers, and on the notion of maternal neglect'. ${ }^{48}$ However, the approach employed for this research on women says much about contemporary attitudes towards gender, 'ways of coping' and psychological illness. The investigative framework was notably different to that 
applied to the seminars, symposia and enquiries into drinking problems in men. To begin with, the group of professionals invited to contribute to discussions included sociologists and marriage guidance counsellors in addition to clinicians and members of the criminal justice system. ${ }^{49}$ Subsequently, specific areas for research included: the role of femininity; recent changes in women's social role; the relationship between drinking and marriage; and how conditioning, upbringing and consequent life expectations might influence drinking. In many discussions, the onset of drinking was noted to be triggered by marital breakdown, in contrast to the assumption that alcoholism in men was likely to lead to divorce. Research questionnaires distributed via staff to patients at treatment centres included explicit questions such as: Why did your drinking become a problem? Do you think that being a woman makes a difference to your drinking problem? Was depression a factor in your drinking? ${ }^{50}$ Staff working at treatment centres were asked specifically about factors that might be unique to women in patient case histories, referral patterns and treatment methods.

Contributors to the CCA's project observed that women were more likely to be labelled as 'depressive', with the alcoholism treated as a secondary disease, if it was diagnosed at all. ${ }^{51}$ Hospital doctors and GPs were more likely to diagnose psychoneurosis to shield a woman from the stigma of alcoholism. Because of this propensity to be diagnosed as 'depressed' and not 'alcoholic', women were subsequently more likely to appear in statistics for psychiatric referral and for treatment with psychotropic drugs. The effects of menstruation, menopause and hysterectomy were explicitly noted to be factors that could influence the onset of drinking, and attention was also paid to possible problems associated with homosexuality, sexual identity and loneliness. These points of reference were in stark contrast to those that emerged in debates about male alcoholics, none of which explored what might be unique about being a 'man' in relation to drinking. Conclusions from this research indeed suggested that women reported drinking when life 'got them down' or when they were 'restless and tense', because it helped them 'forget their worries'. ${ }^{52}$ In psychiatric settings, 'marital discord and domestic stress' were specifically observed as 'precipitating factors for hospitalisation in women', whereas alcoholism was less likely to result in a man being referred for psychiatric assessment at all. ${ }^{53}$

These findings were mirrored in a research paper written by a Scottish psychiatrist, A. B. Sclare, who observed that alcohol problems in women could be correlated specifically to environmental factors related to employment or domestic stress. ${ }^{54}$ Personal testimonies from men, in 
contrast, suggest that they were not comfortable with reflective analysis of their feelings or their situation. One recovering male alcoholic for example recalled: "The question I am often asked is "do you know what caused your drinking?"' to which he added, 'I am not able to isolate any particular cause or causes in myself . . . I am drawn to the conclusion that the most likely hypothesis is that I was conceived on the back of a brewer's dray. ${ }^{\prime 55}$ The CCA's enquiry into female alcoholics thus focused not only on dealing with the social consequences of alcohol abuse, but instead included a set of research questions that were much more likely to identify social, cultural and economic factors that prompted problem drinking.

\section{General practice}

Inevitably, some patients with alcohol problems presented in primary care. However, GPs were primarily concerned with how to diagnose the problem and deal with sickness certification and focused less upon finding out why their patients might drink in the first place. ${ }^{56}$ Many felt that there was so much stigma surrounding alcoholism they were justified in falsifying certificates when a true diagnosis might result in patients losing their job. Glatt conceded that hospital doctors were inclined to do the same thing. ${ }^{57}$ Correspondence from the Rowntree Trust Steering Group on Alcohol also suggests that GPs felt 'services on the NHS were so inadequate that many h[ad] decided not to waste their own time or that of their patients by attempting further use of them'. ${ }^{58}$ GPs, reflecting on their time in practice, confirmed the general picture that alcoholic patients were usually male and that they would usually present with some kind of somatic disorder that would indicate an alcohol habit. Alternatively, their wives would make a visit to the family doctor to report the problem. ${ }^{59}$ Griffith Edward warned GPs that the alcoholic often came into the surgery asking for something for 'bad nerves' or something for 'his stomach', concluding that abnormal drinking may in fact cause, precipitate, imitate or be secondary to every known psychiatric syndrome. ${ }^{60}$

There were important regional differences in the incidence of alcohol abuse, and the characteristics of presentation also varied depending on social class. Although it was eventually determined that Parr's estimate of the numbers of alcoholics nationally was much too low, his study of alcoholism in general practice nonetheless highlighted some distinct regional trends in male drinking. Overall estimates for the south west of England, for example, were relatively low. However, numbers of male alcoholics in the region were particularly high, followed closely by high numbers of 
male alcoholics in the north of England and the Midlands. ${ }^{61}$ Cider drinking among west-country farm labourers resulted in significant alcohol problems that were reflected in Parr's statistics. Personal accounts from GPs who responded to his research questionnaires provided evidence that farm workers regularly drank 'a gallon a day' and this habit would often continue for the duration of their employment. Similar problems were described in the oral histories of retired physicians who had spent their careers in general practice working in Devon and Somerset. One doctor from east Devon, whose practice list consisted largely of farmers and their families, recalled that cider drinking was a 'significant problem', particularly during harvest time. He felt that it was also often related to depression but that it was very difficult to decipher which came first: the depression or the drinking. ${ }^{62}$ Professional journals that focused specifically on alcoholism were able to identify a number of other occupations in which individuals might be vulnerable to over-drinking. Concern was directed in particular towards executive workers who drank alcohol socially as part of their role and those with jobs in the hospitality trade where alcohol was widely available. Other types of employment that allowed abuse to go undetected were also noted. Sickness absence among casual labourers, for example, might go undetected where workers could simply resume work when they had recovered from a drinking bout. ${ }^{63}$ The incidence of alcohol abuse among fishermen had also been a longstanding concern. A retrospective study of alcoholism among Scottish fishermen between 1966 and 1970 suggested that men working in this trade were 'about six times as likely as other men to die of cirrhosis of the liver and were also more prone to peptic ulceration' ${ }^{64}$ It was once again not clear from reports whether or not fishermen drank due to the unique strains of a life at sea, or whether the job attracted 'unusual men' who already had an increased risk of alcoholism. ${ }^{65}$

For GPs dealing with alcoholism in their community, there was a clear distinction between working-class and executive 'habits'. A common theme among interviews was the working-class culture in which men were paid on a Friday, gave their wives 'housekeeping' money, but then spent the rest of their wages on alcohol over the weekend - a practice described pertinently by one GP as 'brickies on blinders' ${ }^{66}$ This culture may account for high numbers of men affected by alcohol abuse in the Midlands and the north of England where manufacturing industry, building and mining predominated. As another doctor recalled:

The culture of the working-class man was, he came, he did a heavy job, which was physically demanding, he sweated a lot, lost a lot of 
fluid, and the culture was, he came home, his wife put the meal on the table, and then off he went to the pub, night after night, to put in lots of beer ... and working men, if you look at the beer consumption, it was absolutely enormous, and it was mostly male. And the pubs were male in those days. ${ }^{67}$

One female GP whose patient list included men who worked for Smithfield and Billingsgate markets in the City of London, recalled that it was difficult to challenge patients about how much they were drinking because 'the norm was very high'. White men in the East End, she recalled, were 'doing it day in, day out'. But in many cases 'they held a job, for a lot of them they managed their life perfectly well, but boy, were they drinking heavily, and were they damaging their health'. ${ }^{68}$ Others pointed out that age was a significant factor for men in socially deprived areas; many older men had serious health problems; co-morbidity and alcoholism was 'a big, big thing in east London'. ${ }^{69}$ Alcoholism, according to one doctor, was very much associated with depressive illness and other psychiatric conditions, complicated further by the fact that older, alcoholic white men with other health conditions tended also to be non-compliant with their medication. ${ }^{70}$

The general consensus among doctors was that alcohol abuse among professional men was perhaps no less common, but that they 'hid it very well' until the problem deteriorated beyond a certain point. ${ }^{71}$ Professionals and semi-professionals were more acutely concerned that their employers did not find out about their alcoholism for fear that they would ultimately lose their jobs. This presented GPs with a dilemma when faced with what diagnosis they should place on the sickness certificate. One doctor remarked:

They would actually say 'Can you put something else down?' So I, I'd say, 'Well how about stress-related?' And they were happy to accept that. Even though if they hadn't been alcoholic they wouldn't have, they were quite happy to, I used to agree with them . . .'make it stress-related, but you and I know that it's an alcohol problem' ${ }^{72}$

David Palmer, when interviewed, agreed that the problem went 'right the way up' the social scale, but that 'the drink [was] different. They drank scotches and gins and things'. Ultimately, he added, whether the men were white- or blue-collar workers, they all drank for 'escapism'. ${ }^{73}$ Alcoholism did not respect class, profession or lifestyle, as one other family doctor pointed out: a church-warden patient of his was 
once found to be behaving strangely, falling asleep in his car and at parish council meetings. They discovered he was stealing the communion wine at about the same time that his wife discovered 'a bottle of whisky in a wellington boot in the garage'. Once again, this doctor felt that the patient's alcoholism had 'probably concealed a degree of depression' ${ }^{74}$

There was little doubt among GPs reflecting on their time in general practice, that the over-use of alcohol was commonly used among men as a coping mechanism. ${ }^{75}$ As was evident in Chapter 2, there was also a general consensus among them that men tended to present with psychosomatic symptoms that were more 'acceptable' and less stigmatising. Sarah Hall, who had a particular interest in the psychological dimension of disease, noted that in her London practice alcohol presented in many ways, but that dyspepsia was one of the most common:

So, with the dyspepsia, you know, probably, the first thing you thought of is alcohol. And, if you had really ruled that out, you know, then you began to wonder about, whether there was also a psychological element to it. But simply, the person who was always taking Monday and Tuesday off, and so wanting certification. And of course, often they would also come and say they'd got back pain. And, so, some of the back pains were actually problems with alcohol, but they didn't want to admit that, so they just turned it into back pain. ${ }^{76}$

Indeed, employers were warned by alcohol experts to be suspicious of repeated sickness certificates for gastritis, signs of irritability, decreased performance and poor time-keeping. ${ }^{77}$ They were also advised to be alert to absences on Monday mornings, particularly 'if a wife phoned in', since this might indicate a weekend of heavy drinking. ${ }^{78}$ Such concerns did not go entirely unnoticed by the media, as occasional articles were released in the press highlighting the issue of sickness absence due to alcohol. One headline in 1970 warned that 'Monday is hangover day for British industry', and claimed that 'a quarter of a million men in Britain will be off sick today, when all they have is a bad hangover'. ${ }^{79}$ Another news item in the Daily Express described the problem as 'a secret illness' and as 'the complaint that nobody wants to talk about'. 80

Not all GPs were as perceptive as Hall when it came to recognising somatic symptoms caused by alcohol abuse. As Glatt pointed out in 1960, doctors were 'not well-trained to suspect or diagnose the 
condition in its early phases' and in many cases 'doctors and alcoholics [did] not care a great deal for crossing each other's path' ${ }^{81}$ The personality of individual doctors certainly influenced their patterns of diagnosis. In a lengthy article on this topic that covered numerous research studies, H. J. Walton, a psychiatrist from the University of Edinburgh, found that a substantial proportion of both medical students and experienced GPs reacted unfavourably to patients presenting with psychosomatic disorders without serious organic disease. ${ }^{82}$ Although most doctors fully accepted a responsibility towards such patients, those whom Walton described as 'physically-minded' as opposed to 'psychologicallyminded' found alcoholic patients to be 'not acceptable' and described them as a 'clinical burden'. ${ }^{83}$ An enquiry into GP's opinions about alcoholism also found that although an increasing number of doctors viewed the condition as an illness, 'a disturbing minority still [thought] of it in terms of moral weakness or weakness of willpower, or sin and vice'.$^{84}$ This, the author observed, was worth noting precisely because such opinions were likely to be reflected in attitudes towards, and management of alcoholic patients. ${ }^{85}$ Concerns about the difficulties associated with understanding alcoholism and alcohol-related behaviour prompted a sociologist from the Addiction Research Unit at the Institute of Psychiatry to remind the medical profession that, although the over-use of alcohol resulted in, on the one hand a 'biochemical and physiological state', on the other hand, the function of 'noticing, recognising, responding to and treating' it should be seen within the context of both personal and societal 'beliefs' about the condition - and wider culturally held values about such issues as personal responsibility and 'appropriate' behaviour. Thus, whatever the medical basis of the condition, much of the decision-making process about diagnosis and treatment depended upon 'explicitly social considerations'. ${ }^{86}$ Given doctors' paucity of training in psychological medicine, the lack of postgraduate training for general practice, and the broader stigma and indifference towards alcoholism, it is perhaps not surprising that men who self-medicated for emotional problems were reluctant to seek help from family doctors and were often diagnosed incorrectly when they did so.

\section{Reflections}

In an article published in the British Journal of Addiction in 1963, Herbert Berger, an American physician, lamented existing approaches towards alcohol abuse. ${ }^{87} \mathrm{He}$ had deliberately changed the title of his paper from 'The treatment of alcoholism' to 'The prevention of alcoholism', 
arguing that the word 'treatment' should be 'dropped' from its prominent place in discussions. ${ }^{88}$ Berger recommended instead, a 'philosophy of alcoholism' in which 'causative factors' should be central to investigations. ${ }^{89}$ His core argument was that alcohol was a 'secondary aetiology' - the prime cause being 'some difficulty' making it 'impossible for the patient to cope with the vicissitudes of his environment' ${ }^{90}$ Berger reminded the medical profession that the need for 'escape' was a normal human attribute and that humans in every culture had practised emotional release from daily frustrations. In this time and place, he noted, 'making the environment more tolerable' included drinking alcohol as medication for the relief of depression and 'as a lubricant to forget one's troubles ... to blur one's accurate observation of stark reality'. Failing to focus on the environmental causes of alcoholism, he warned, would result simply in 'shifting addictions from one material to another'.$^{91}$ In his paper, Berger also criticised AA for its practice of leaving alcoholics to 'hit rock bottom', arguing that in no other speciality of medicine did physicians 'wait until the patient has practically succumbed to a disease before attempting to effect a cure' ${ }^{92}$ Berger thus broadly urged both the medical profession and AA to do more in terms of preventative medicine, concluding that 'no man is an island' and the entire community was needed to attend to the problem. ${ }^{93}$

Berger's comments were expressly relevant to those working in the alcohol arena in Britain. Speaking in 1963 at the annual dinner of the Society for Study of Addiction, Kenneth Robinson MP, acknowledged that there was less than good provision on all fronts in Britain compared with America and some other countries. ${ }^{94}$ Commentators noted with regularity that approaches to alcoholism in other countries such as America, Norway and Sweden more readily provided initiatives to help alcoholics that included the use of psychiatrists, psychologists and social workers to explore the social and cultural aspects of the disease. ${ }^{95}$ Countries where the temperance movement had previously asserted more influence, despite the divisions this caused, spoke more candidly about alcohol abuse and its problems and were more open to exploring alternative dimensions of the disease. As Selden Bacon, the Director of Alcohol Studies at Rutgers University noted, by the 1960s, the rigid structures of the temperance camp, the anti-temperance camp and the 'avoiders' (who were more opposed to the conflict than to alcohol itself) had begun to lose their power. The resulting interchange of ideas emphasised tested knowledge and an evidence-based approach. Furthermore, as Lord Soper pointed out in the House of Lords debate in 1965, Canada, Australia, New Zealand and Australia received 'a great deal of 
government assistance' for alcohol research, and in Scandinavian countries, where there was a state monopoly of the manufacture and sale of alcohol, a proportion of the profits were ploughed back into research and education. ${ }^{96}$ Consequently, as the previous chapter demonstrated, industrial employers were more likely to provide programmes providing assistance to alcoholic workers. The contrast in Britain was stark: there was widespread denial among industry leaders and within the Ministry of Health, while the state benefited from large revenues from the duty on alcoholic beverages. It is notable that, in Britain during the 1970s, when concern was eventually raised about female alcoholism, research questions were constructed around a more productive framework, less focused on aspects of treatment and diagnosis, and more upon what it might be about the female role that caused women to abuse alcohol. Betsy Thom has argued that the feminist movement of the 1960s was instrumental in this respect, since it had begun to frame women's health issues in political, social and economic terms. It thus provided the ideological motivation for explanations of women's use and misuse of alcohol, emphasising the social and psychological context of drinking. ${ }^{97}$ As this book has illustrated, the men's movement in Britain was less influential and there were no prominent initiatives actively questioning the male role and its impact on men's wellbeing.

The problem was exacerbated further by the fact that manufacturers of alcoholic beverages directly targeted men in their advertising campaigns, which promoted drinking as not only a pleasurable pastime, but also increasingly as a way to relieve stress. During the 1950s, these advertisements appeared widely in daily newspapers and also in publications directed exclusively at men, such as Lilliput and Men Only. Whisky adverts even claimed that alcohol had 'health-giving' properties: 'a White Horse toddy at bedtime', for example was supposed to 'promote warmth and glow of wellbeing' while 'disarming the threat of colds or influenza' ${ }^{98}$ The manufacturers of the fortified wine, Dubonnet, stated that their drink was an effective 'tranquilliser' and that 'at no time does it affect the liver', despite its alcohol by volume (ABV) content of over 14 per cent. ${ }^{99}$ During the mid-1960s, alcohol often featured in the advertising matter in the Journal of the College of General Practitioners. Guinness in particular was promoted with regularity for consumption both by patients and doctors. One advert featured a cartoon of a man in sports vest and shorts, jogging - while at the same time drinking a pint of Guinness. The caption read: 'Dear Doctor, I have taken Guinness for seven days running and how much better I feel. ${ }^{100}$ Another, aiming directly to entice medical professionals, and picturing a cartoon of an 
exhausted-looking doctor, suggested that 'When you've been worked off your feet . . . Relax with a Guinness. ${ }^{101}$ Concerns about trends in advertising developed from the 1960s as manufacturers increasingly drew upon sexualised images to promote their products. Lord Soper condemned the alcohol adverts as 'unscrupulous', arguing that they invited young people - if they wanted to be virile - to be constantly taking in alcohol. ${ }^{102}$ An article in The Times in 1979, urged the alcohol industry to be more responsible, since it had caused trouble confusing 'good' things like holidays and sport with drinking, while being mendacious about the true merits of alcohol. ${ }^{103}$ Lemle and Mishkind noted in research published in 1989, that through the second half of the twentieth century social drinking increasingly became a primary cultural symbol of 'manliness'. ${ }^{104}$ Heavy drinking symbolised greater masculinity than lighter drinking, and the more a man tolerated his alcohol, the more manly he was deemed. ${ }^{105}$

Accounts from a Mass Observation investigation into public houses and drinking confirm indeed that working-class men were inclined to drink to appear 'tough' and to fit in with their peers. The cultural association between alcohol consumption and masculinity was clearly evident in the words of one respondent who claimed: 'My reason for drinking beer is to appear tough. I heartily detest the stuff, but what would my pals think if I refused? They would call me a cissy. ${ }^{\prime 106}$ Another declared that he only went into the pub with his friends 'for the sake of their company'. ${ }^{107}$ Many noted the apparent health-giving properties of alcohol, listing its 'good effect on appetite', and its 'laxative and sleepinducing effects' as reasons for drinking. These reactions, the authors of the study noted, indeed reflected the themes promoted heavily in brewers' advertising. ${ }^{108}$ Beer-drinking was also widely associated with increased sexual performance. One pub-drinker declared that 'if [he got] three pints down [him]', he 'was able to have sexual intercourse with the maximum of efficiency and when he woke up in the morning he was able to repeat the process with the utmost satisfaction' ${ }^{109}$ This Mass Observation study was primarily of working-class beer drinkers; however, the publicans who were interviewed observed that spirit drinkers tended to be businessmen, who were 'hard-pressed by work or financial matters, fall[ing] to spirits as a quick consolation to forget matters'. ${ }^{110}$ The authors also concluded that a large amount of wine and spirits was being consumed at home by the middle class. ${ }^{111}$

From the accounts of physicians, the growing concerns of those working in the alcohol arena and in industry, it is clear that for men, drinking alcohol was a common means of escapism. In the workplace 
and during leisure activities, ideas about the degree to which it was seen as appropriate to admit to emotional difficulties discouraged men from seeking help for problems both at work and at home. On a rudimentary level, men appeared unable or unwilling to look introspectively at the cause of their problems. These issues were compounded further by the dominance of the disease theory during the 1950s and 1960s, which assumed the alcoholic to be in the minority, diverting attention away from broader consumption levels and social factors in causation. ${ }^{112}$ During the post-war period, GPs and hospital physicians were also poorly trained in psychological medicine and, until the late 1970s, were usually male and therefore affected by the same difficulties when challenged to be reflective or emotionally expressive. Many unwittingly colluded with stereotypical views about femininity and masculinity, providing psychiatric diagnoses for women and somatic diagnoses for men. Quite often, both the male patient and the doctor were satisfied with a somatic diagnosis and looked no further.

Except where otherwise noted, this work is licensed under a Creative Commons Attribution 3.0 Unported License. To view a copy of this license, visit http://creativecommons.org/licenses/by/3.0/ 